\title{
Helium Ion Nanomachining in Membranes and Bulk Substrates
}

\author{
E.M. Mutunga, ${ }^{1}$ A.E. Vladár, ${ }^{2}$ L.A. Stern ${ }^{3}$ and K.L. Klein, ${ }^{1,2}$ \\ ${ }^{1}$ University of the District of Columbia, 4200 Connecticut Ave NW, Washington, DC 20008 \\ ${ }^{2}$ National Institute of Standards and Technology, 100 Bureau Drive, Gaithersburg, MD 20899 \\ ${ }^{3}$ Carl Zeiss Microscopy LLC, One Corporation Way, Peabody, MA 02169
}

Machining materials on the nanometer scale has many applications ranging from nanoelectronics and integrated circuit editing [1] to the creation of nanopore sensors [2] and optical devices [3]. The helium ion microscope (HIM), with a typical probe size of less than $1 \mathrm{~nm}$, offers a unique method for nanofabrication at a s cale currently unattainable by conventional Gabased focused ion beam (FIB) processing. The HIM's gas field ion source, produced with $\mathrm{He}^{+}$, results in a smaller interaction volume near the sample surface and lower sputter yield as compared to heavier ions [4]. While gallium atoms deposit most of their kinetic energy near the surface (making $\mathrm{Ga}^{+}$highly effective at bulk milling), the resulting interaction volume yields feature sizes much larger than the beam diameter. Given the long range of $\mathrm{He}^{+}$in most materials [5], the sputtering rate is lower than for a gallium beam; however, this means that the sample interactions don't spread the beam as quickly and sputtering events are much more likely to occur close to the beam axis (enabling smaller milling feature sizes).

Previous work has shown that some substrates such as $\mathrm{SiO}_{2}$ and $\mathrm{Au}$ readily mill under the beam while other substrates such as $\mathrm{Si}$ and $\mathrm{SiC}$ exhibit a drastically different "swelling" behavior under high irradiation dose as shown in Fig. 1(a) [6]. HIM-machined feature sizes of $5 \mathrm{~nm}$ or smaller have been demonstrated in thin membranes [2,3,7], however it remains to be seen how mill $\mathrm{r}$ ates and attainable feature quality depend upon the film thickness and milling conditions; nor has bulk milling been studied in detail. Contrary to earlier work [7], a recent publication indicates that backside or transmission milling in membranes with thicknesses less than the ion range is a significant factor leading to nonlinearity in the mill rate for silicon nitride membranes [8].

As with any FIB technique, there are trade-offs between probe size and current, i.e., machining accuracy and minimizing the pattern write time and/or drift. It is also important to use the minimum dose necessary to achieve the desired result, thereby producing the smallest features with the least substrate damage. We have observed that surface contamination significantly affects the milling of gold. The low sputter yield with $\mathrm{He}^{+}$(about 2 or ders of magnitude lower than with $\mathrm{Ga}^{+}$) presumably gives contaminants time to diffuse to the milling site. Figure $1(\mathrm{~b}, \mathrm{c})$ illustrates the necessity of in-situ plasma cleaning of the chamber and sample to achieve contamination-free machining. In Figure 2(a), we demonstrate how the He ion beam can be used to machine nano-to-micrometer-sized trenches, here less than $10 \mathrm{~nm}$ wide at a $30 \mathrm{~nm}$ pitch. Additionally, in Figure 2(b,c) we show that $14 \mathrm{~nm}$ holes can be milled through an approximately $100 \mathrm{~nm}$ thick Au foil and that by reducing the film thickness, holes of half that diameter could be produced under the same conditions but with $10 \%$ of the dose. In this paper we will investigate milling of $\mathrm{Au}$ and other substrates as a function of thickness and beam parameters and compare this to ion beam scattering models [5] to better understand the machining process in thin films and bulk substrates. 
[1] S. Tan, et al., J. Vac. Sci. Technol. B 29 (2011), 06F604-1.

[2] J. Yang, et al., Nanotechnology 22, (2011), 285310.

[3] L. Scipioni, Carl Zeiss, Adv. Mat. Char.Workshop, U. Illinois, (2012).

[4] J.A. Notte, Microscopy Today 20, 16 (2012), p. 16.

[5] J.F. Zeigler, et al., SRIM-2012.03 modeling freeware available at www.srim.org (2012).

[6] K.L. Klein, et al., Microscopy and Microanal. Vol. 18, S2 (2012), p. 830.

[7] L. Scipioni, et al., J. Vac. Sci. Technol. B 28, C6P18 (2010).

[8] M.M. Marshall, J. Yang, and A.R. Hall, Scanning 34, (2012), p. 101.

[9] The authors would like to thank Carl Zeiss Microscopy for their support.

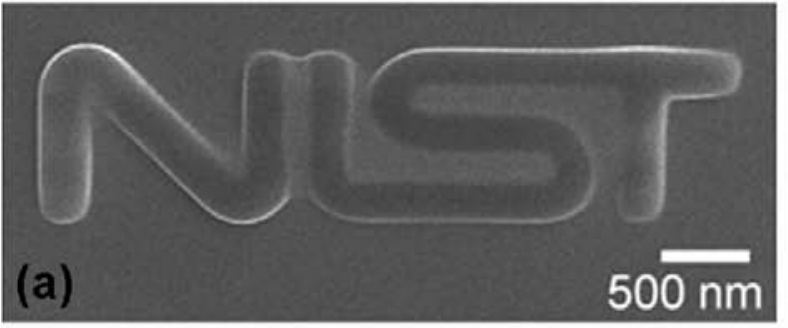

\section{(b)}
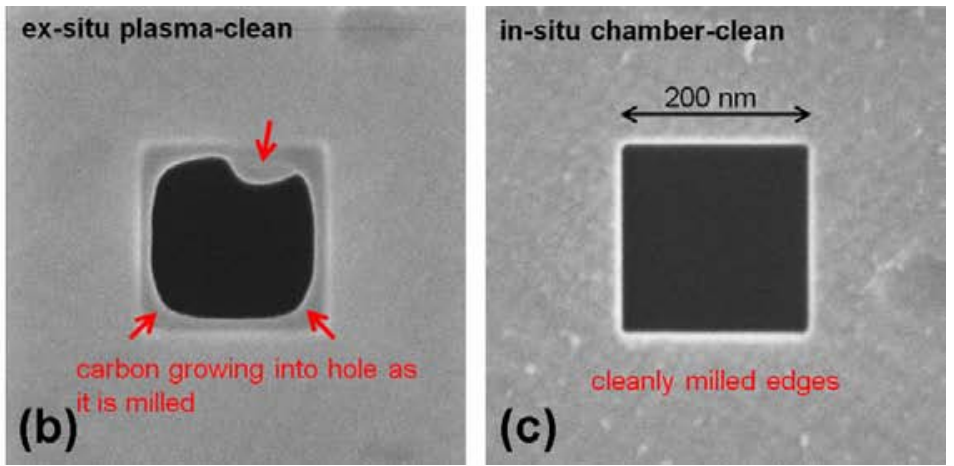

Figure 1. Helium ion micrographs of (a) helium swelling in a silicon substrate (b,c) $200 \mathrm{~nm}$ square features milled through a $\sim 100$-nm-thick gold foil. In (b) the sample was oxygen plasma cleaned exsitu, while in (c) the sample and chamber were oxygen plasma cleaned in-situ. Scan conditions: $35 \mathrm{keV}$, $4 \mathrm{pA}, 1 \mu \mathrm{s}$ dwell time.
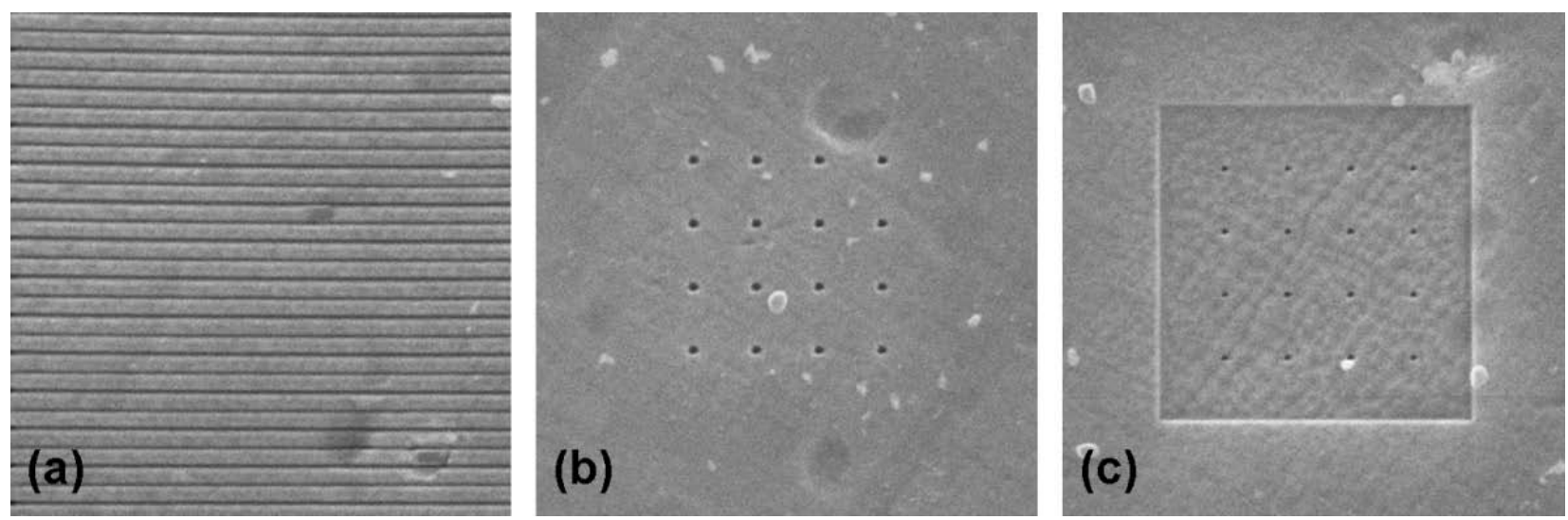

Figure 2. Helium ion micrographs patterns in a 100-nm-thick gold foil. (a) $<10 \mathrm{~nm}$ milled lines at 30 nm pitch. A $14 \mathrm{~nm}$ (b) and a $7 \mathrm{~nm}$ (c) hole array produced with 1 second and with a 0.1 second dwell time respectively, for (c) the foil was first thinned by He ion milling over a $500 \mathrm{~nm}$ by $500 \mathrm{~nm}$ area. Beam conditions: $35 \mathrm{keV}, 4 \mathrm{pA}$. All are $800 \mathrm{~nm}$ field-of-view images. 